\title{
Does nutritionist review of a self-administered food frequency questionnaire improve data quality?
}

\author{
Bette J Caan ${ }^{1, *}$, Elaine Lanza ${ }^{2}$, Arthur Schatzkin ${ }^{3}$, Ashley O Coates ${ }^{1}$, Brenda K Brewer ${ }^{4}$, \\ Martha L Slattery ${ }^{5}$, James R Marshall ${ }^{6}$ and Abby Bloch ${ }^{7}$ \\ 'Kaiser Permanente Medical Care Program of Northern California, Division of Research, 3505 Broadway, \\ Oakland, CA 9461 1, USA: ${ }^{2}$ National Cancer Institute, Cancer Prevention Studies Branch, 6006 Executive Blvd, \\ Suite 311 , Rockville, MD 20852, USA: ${ }^{3}$ National Cancer Institute, Nutrition Epidemiology Branch, \\ 6130 Executive Blvd, Room 211 , Rockville, MD 20852, USA: ${ }^{4}$ Westat, 1650 Research Blvd, Rockville, \\ MD 20850-3129, USA: ${ }^{5}$ School of Medicine, Division of Public Health Sciences, University of Utah, \\ 546 Chipeta Way, Suite 1 100, Salt Lake City, UT 84108, USA: ${ }^{6}$ Arizona Cancer Center, \\ 1515 Campbell Avenue, Tucson, AZ 85724, USA: ${ }^{7340}$ East 64th Street, New York, NY 10021, USA
}

Submitted 20 January 1999: Accepted 1 June 1999

\begin{abstract}
Objective: This study sought to evaluate the benefit of utilizing a nutritionist review of a self-administered food frequency questionnaire (FFQ), to determine whether accuracy could be improved beyond that produced by the self-administered questionnaire alone.

Design: Participants randomized into a dietary intervention trial completed both a FFQ and a 4-day food record (FR) at baseline before entry into the intervention. The FFQ was self-administered, photocopied and then reviewed by a nutritionist who used additional probes to help complete the questionnaire. Both the versions before nutritionist review and after nutritionist review - were individually compared on specific nutrients to the FR by means, correlations and per cent agreement into quintiles.

Settings and subjects: Three hundred and twenty-four people, a subset of participants from the Polyp Prevention Trial - a randomized controlled trial examining the effect of a low-fat, high-fibre, high fruit and vegetable dietary pattern on the recurrence of adenomatous polyps - were recruited from clinical centres at the University of Utah, University of Buffalo, Memorial Sloan Kettering Cancer Center in New York and Kaiser Permanente Medical Program in Oakland.

Results: Reviewing the FFQ increased correlations with the FR for every nutrient, and per cent agreement into quintiles for all nutrients except calcium. Energy was underestimated in both versions of the FFQ but to a lesser degree in the version with review.

Conclusions: One must further evaluate whether the increases seen with nutritionist review of the FFQ will enhance our ability to predict diet-disease relationships and whether it is cost-effective when participant burden and money spent utilizing trained personnel are considered.
\end{abstract}

Keywords
Food frequency
Diet methods
Validation
Dietary intake
The association between diet and chronic diseases is of great interest. In 1988, the US Surgeon General claimed that $68 \%$ of all deaths in the United States resulted from diet-related diseases ${ }^{1}$. As a result, much effort has been spent on the development of dietary assessment instruments that are easy to administer, cost-efficient and valid.

For epidemiological studies of large populations, the FFQ is the most commonly used dietary assessment method $^{2}$. FFQs, which can be self-administered or interviewer-administered, typically ask the respondent to report usual frequency of consumption of each food from a list of foods for a specified time period. Many FFQs also include questions on usual portion size and a few general questions regarding cooking methods and additions to foods. The foods listed on the FFQ address specific foods consumed by the population under study or food groups of interest. FFQs measure current or past intake.

The self-administered FFQ requires a literate population; it may also result in inconsistent interpretation and lower than desired response and completion rates, each of which might compromise the validity of the data. Validity studies of the self-administered FFQ, 
which have generally compared the FFQ with food records or serial 24-hour diet recalls, have produced correlation coefficients that range between 0.24 and $0.81^{3-7}$.

A more costly labour-intensive method, the interviewer-administered history or $\mathrm{FFQ}^{8}$, was concluded to be, in a 1987 methodological survey completed by the USDA, among the most effective methods for assessing dietary intakes of groups?

Comparing results from validation studies of selfadministered FFQs and results from validation studies of interviewer-administered diet histories or FFQs is difficult since the individual studies vary by design and few studies directly compare the two methods. However, correlations from validation studies for interviewer-administered instruments ranged from 0.52 to $0.80^{10-12}$, with many fewer of the studies producing correlations below 0.50 as seen in the validation studies of self-administered questionnaires.

In one study, which directly compared intervieweradministered FFQs to self-administered FFQs and attempted to estimate intake 10-15 years in the past, data from the self-administered FFQs produced lower correlations compared with reference data ${ }^{13}$ than data from the interviewer-administered FFQ. A second study, which compared a self-administered diet questionnaire and an interviewer-administered diet history to 7-day FRs found the two questionnaire methods to be comparable for most nutrients. Average correlations between FRs and self-administered questionnaires were slightly higher than between FRs and interviewer-administered questionnaires ${ }^{14}$. A third study ${ }^{15}$ compared a telephone-administered FFQ to self-administered FFQs and found rankings from telephone-administered FFQs much closer to in-person interviewer-administered FFQs than those from self-administered FFQs.

Since study subjects may often be available in person, but utilizing an interviewer-administered dietary instrument is time-consuming and costly, this study sought to evaluate the benefit of utilizing a hybrid approach: a brief review of a self-administered FFQ by a trained nutritionist, to determine whether the accuracy could be improved beyond that produced by the self-administered FFQ alone.

\section{Methods}

Three hundred and twenty-four people, a subset of participants from the Polyp Prevention Trial - a randomized controlled trial examining the effect of a low-fat, high-fibre, high fruit and vegetable dietary pattern on the recurrence of adenomatous polyps completed both baseline 4-day FRs and FFQs. The study population consisted of all participants who were randomized in 1993 in four of the seven clinics invited to participate in the ancillary study. Clinic participation was based on the presence of a scientific investigator interested in nutrition methodology.

Food records were collected at baseline for four consecutive days (Sunday to Wednesday). Participants were instructed how to complete the FRs in a standardized manner from an instructional videotape during the first screening visit. The completed FRs were returned at the second screening visit and a nutritionist retrieved additional information in order to properly code records for analysis. The FRs were analysed using the Minnesota Nutrition Data System (NDS) software, developed by the Nutrition Coordinating Center, University of Minnesota, Minneapolis (food database version $5 \mathrm{~A}$, nutrient database version 20 ).

The FFQ used in this study, modified from the 97item Block/NCI FFQ ${ }^{16}$, contained 105 items. It included more high-fibre foods, such as dried fruit, high-fibre cereals and legumes, and more low-fat and non-fat foods.

Participants were instructed at the baseline initial screening visit on how to complete the questionnaire, and were asked to recall their intake over the past year. They were asked to complete it at home and return it at their second screening visit, approximately 2 weeks later. The questionnaire was then immediately photocopied (thus retaining a copy of the unedited version) and reviewed with the participant by the same nutritionist who reviewed the FR, probing for foods that may have been inadvertently missed, counting the number of main meals and checking for data completeness. The FFQ was always reviewed after the FR. A standard set of recommended probes were given to each nutritionist to include in their review (see Appendix). For example, the nutritionist asked if they had missed any vegetables either that were not on the list or that they had in only small amounts such as at a salad bar, and whether the salad dressings they reported included those they may have had on sandwiches. Since the study was a dietary intervention trial aimed at decreasing overall fat intake and increasing fruit, vegetable and fibre intake, many of the probes were designed to capture complete information on grains, fruits, vegetables and added or hidden fats. The questionnaires were then edited to reflect changes from the review.

Mean values for nutrients were calculated for the FR, FFQ1 and FFQ2. Mean food group servings were calculated for FFQ1 and FFQ2. Paired $t$-tests were used to test differences between FFQ1 and FFQ2. For comparisons of FR to FFQ, data were natural logtransformed. Energy, percentage energy as fat, protein or carbohydrate, and energy-adjusted nutrients (residual method ${ }^{17}$ ) were compared separately to both FFQ1 and FFQ2, using Pearson correlations and per cent agreement within \pm 1 quintile. 
Table 1 Means, correlations and per cent agreement for classifications into the same $( \pm 1)$ quintile for nutrients (per day) from food records (FR) and food frequency questionnaires, unedited (FFQ1) and after nutritionist review (FFQ2) $(n=324)$

\begin{tabular}{|c|c|c|c|c|c|c|c|}
\hline \multirow[b]{2}{*}{ Nutrients (day) } & \multicolumn{3}{|c|}{ Mean } & \multicolumn{2}{|c|}{$\begin{array}{c}\text { Correlation* }{ }^{*} \\
\text { between FR and }\end{array}$} & \multirow{2}{*}{$\begin{array}{c}\% \text { agreement for } \\
\text { FR and FFQ1 } \\
( \pm 1 \text { quintile }) \dagger\end{array}$} & \multirow{2}{*}{$\begin{array}{l}\text { \% agreement for } \\
\text { FR and FFQ2 } \\
( \pm 1 \text { quintile }) \dagger\end{array}$} \\
\hline & $\mathrm{FR}$ & FFQ1 & FFQ2 & FFQ1 & FFQ2 & & \\
\hline Energy (kcal) & 2034 & 1777 & $1913 \ddagger$ & 0.41 & 0.53 & 63.6 & 68.8 \\
\hline Fat (q) & 74.9 & 70.9 & $77.1 \neq$ & 0.61 & 0.65 & 72.2 & 78.7 \\
\hline$\%$ energy as fat & 32.6 & 34.9 & $35.7 \ddagger$ & 0.57 & 0.64 & 65.8 & 70.4 \\
\hline Saturated fatty acids (g) & 25.3 & 24.8 & $26.7 \ddagger$ & 0.59 & 0.64 & 72.5 & 75.0 \\
\hline Oleic acid $(\mathrm{g})$ & 25.8 & 25.9 & $28.2 \ddagger$ & 0.55 & 0.59 & 72.2 & 74.1 \\
\hline Linoleic acid (g) & 13.7 & 12.8 & $14.0 \ddagger$ & 0.42 & 0.47 & 67.3 & 71.3 \\
\hline Protein $(\mathrm{g})$ & 80.9 & 71.7 & $78.7 \ddagger$ & 0.36 & 0.40 & 61.4 & 69.5 \\
\hline$\%$ energy as protein & 16.2 & 16.3 & $16.7 \ddagger$ & 0.41 & 0.43 & 66.7 & 65.6 \\
\hline Carbohydrate $(\mathrm{g})$ & 258 & 206.9 & $219 \neq$ & 0.59 & 0.65 & 74.7 & 80.3 \\
\hline$\%$ energy as carbohydrate & 51.0 & 47.2 & $46.1 \ddagger$ & 0.57 & 0.64 & 75.6 & 80.6 \\
\hline Dietary fibre $(\mathrm{g})$ & 19.5 & 17.4 & $17.9^{\top}$ & 0.62 & 0.65 & 75.9 & 76.2 \\
\hline Calcium (mg) & 801 & 806 & $859 \neq$ & 0.55 & 0.62 & 77.5 & 77.2 \\
\hline Beta-carotene $(\mu \mathrm{g})$ & 4555 & 3978 & $4464 \ddagger$ & 0.47 & 0.49 & 67.6 & 70.1 \\
\hline Vitamin C (mg) & 129 & 134 & 136 & 0.51 & 0.55 & 70.7 & 74.7 \\
\hline Folate $(\mu \mathrm{g})$ & 326 & 293 & $301 \S$ & 0.46 & 0.52 & 63.9 & 70.1 \\
\hline
\end{tabular}

* Pearson correlations.

†All data are log-transformed. Nutrients are either energy, \% energy as fat, protein or carbohydrate, or energy-adjusted nutrients (residual method).

$\ddagger$ Difference between FFQ1 and FFQ2 is significant at $P<0.01$ level.

$\S$ Difference between FFQ1 and FFQ2 is signficant at $P<0.05$ level.

\section{Results}

The study population was $65.7 \%$ female and $34.2 \%$ male. Slightly over half of them (56.8\%) were 60 years or over. These demographics are very similar to the sex and age distribution of the overall participant population in the Polyp Prevention Trial.

Editing the FFQ significantly increased estimates of all nutrients except dietary fibre and vitamin C. Table 1 demonstrates that while both FFQ1 and FFQ2 underestimate caloric intake, FFQ2 underestimates it to a lesser degree. While absolute fat intake on the FFQ1 is lower than the value from the FR and absolute fat from

Table 2 Means (servings per week) for food groups from the unedited food frequency questionnaire (FFQ1) and after nutritionist review (FFQ2) $(n=324)$

\begin{tabular}{lcc}
\hline & \multicolumn{2}{c}{ Mean } \\
\cline { 2 - 3 } Food groups & FFQ1 & FFQ2 \\
\hline Fruit & 9.3 & 9.0 \\
Juice & 4.4 & 4.4 \\
Vegetables & 15.7 & $16.1^{*}$ \\
Whole grains & 4.9 & $5.3^{*}$ \\
Fibre cereals & 3.8 & 3.6 \\
Fish, chicken & 3.3 & $3.8^{*}$ \\
Beef, pork & 2.7 & $3.1^{*}$ \\
Breakfast meat & 0.9 & 0.9 \\
Hot dogs/lunch meat & 1.7 & $1.9^{\star}$ \\
Eggs & 2.1 & 2.0 \\
Butter, margarine & 7.7 & 7.5 \\
Milk, yogurt & 7.7 & 7.7 \\
Cheese & 1.4 & $1.7^{\star}$ \\
Frozen desserts & 1.6 & $1.9^{*}$ \\
Other desserts & 4.2 & $4.5^{*}$ \\
Alcohol & 3.8 & 3.8 \\
Chips and nuts & 2.3 & $2.7^{*}$ \\
\hline
\end{tabular}

* Difference between FFQ1 and FFQ2 is significant at $P<0.01$ level.
FFQ2 is higher than the FR value, both FFQ1 and FFQ2 seem to overestimate percentage energy as fat compared to the FR. FFQ1 produced protein values lower than those obtained by the FR; editing increased protein values on FFQ2 to a level similar to that obtained from FR, but that resulted in an overestimation of percentage energy from protein on FFQ2. Values for carbohydrates for both FFQ1 and FFQ2 were lower than from the FR and both FFQ1 and FFQ2 underestimated percentage energy as carbohydrates as well. FFQ1 and FFQ2 underestimate dietary fibre, although FFQ2 underestimates less than FFQ1. Fatty acids and calcium are overestimated by FFQ2. Editing the FFQ significantly increased mean intakes in nine out of 17 food groups (Table 2). Editing increased correlations with the FR for every nutrient and per cent agreement into quintiles for all nutrients except calcium and percentage energy as protein (Table 1).

We regressed total fat from FFQ1 and FFQ2 on the FR (data not shown). Both the slopes from FFQ1 and FFQ2 were flattened compared to ideal (1.0), with the slope from FFQ2 slightly less flat and closer to ideal than that from FFQ1, mostly due to those with high intakes underreporting less on FFQ2 then they did on FFQ1.

\section{Discussion}

Review and editing of the FFQ increases means, correlations and per cent agreement into quintiles for energy and the majority of nutrients, suggesting some degree of underreporting in the unedited self-administered questionnaire. In addition, we have demonstrated that tailoring instruments, and/or reviewing or probing, designed to gain accuracy for a 
particular nutrient or food group, could lead to overestimation of that nutrient. In our study, which included the addition of reduced-fat food items to the questionnaire and specific probes to obtain more accurate information on fat intake, the nutritionist review increased energy estimates while simultaneously overestimating absolute fat, resulting in an even greater overestimation of percentage energy from fat than was already seen in FFQ1. An alternative approach, to avoid such a bias, would be to design general probes to be administered with each FFQ that would not vary depending on the specific focus of the study or specific study hypotheses.

One limitation of this study is that we assume that the FR provides an unbiased estimate of truth, which has been recently questioned by Freedman ${ }^{18}$. Another limitation is that the same dietitian reviewed both the FR and FFQ, which could induce some correlation of error. However, this is unlikely since FR retrievals were focused on getting adequate information on listed foods to enable proper coding and analysis, while the review of the FFQ focused on probes for foods that might have been missed or overestimated. Additionally, the increases in correlations observed with FFQ2 were small, thus adding to the likelihood that this bias was not substantial.

This study was not able to answer whether improvements seen with nutritionist review of the FFQ might also be realized through computerized editing mechanisms. Previous research ${ }^{19}$ has demonstrated that items left blank on self-administered FFQs are often foods that people eat rarely or never. As a result a large number of blanks (somewhere in the range of $10-15 \%$ of the total number of items) will not affect the ability of the questionnaire to rank individuals ${ }^{19}$. Further research must evaluate the costeffectiveness of a nutritionist review, compared to alternative means of editing, when the additional time and money spent utilizing trained personnel to review, probe and edit questionnaires is considered. Also, further research must evaluate whether improvements of the degree demonstrated in this study are enough to influence our ability to predict disease, given exposure data that is already prone to large amounts of error.

\section{REFERENCES}

1 US Department of Health and Human Services. The Surgeon General's Report on Nutrition and Health. Publication No. 88-50210. Washington, DC: Government Printing Office, 1988.

2 Thompson FE, Moler JE, Freedman LS, Clifford CK, Stables GJ, Willett WC. Register of dietary assessment calibrationvalidation studies: a status report. Am.J. Clin. Nutr. 1997; 65 (Suppl. 4): S1142-7.

3 Martin LJ, Lockwood GA, Kristal AR, et al. Assessment of a food frequency questionnaire as a screening tool for low fat intakes. Controlled Clin. Trials 1997; 18(3): 241-50.
4 Friis S, Kruger Kjaer S, Stripp C, Overvad K. Reproducibility and relative validity of a self-administered semiquantitative food frequency questionnaire applied to younger women. J. Clin. Epidemiol. 1997; 50(3): 303-11.

5 Pisani P, Faggiano F, Krogh V, Palli D, Vineis P, Berrino F. Relative validity and reproducibility of a food frequency dietary questionnaire for use in the Italian EPIC centres. Int. J. Epidemiol. 1997; 26 (Suppl. 1): S152-60.

6 Van Lierre MJ, Lucas F, Clavel F, Slimani N, Villeminot S. Relative validity and reproducibility of a French diet history questionnaire. Int. J. Epidemiol. 1997; 26 (Suppl. 1): $\mathrm{S} 128-36$.

7 Boering H, Bohlscheid-Thomas S, Voss S, Schneeweiss S, Wahrendorf $J$. The relative validity of vitamin intakes derived from a food frequency questionnaire compared to 24-hour recalls and biological measurements: results from the EPIC pilot study in Germany. European Prospective Investigation into Cancer and Nutrition. Int. J. Epidemiol. 1997; 26 (Suppl. 1): S82-90.

8 Barrett-Connor E. Nutrition epidemiology: how do we know what they ate? Am.J. Clin. Nutr. 1991; 54: S182-7.

9 Morgan KJ, Johnson SR, Rizek RL, Reese R, Stampley GL. Collection of food intake data: an evaluation of methods J. Am. Diet. Assoc. 1987; 87(7): 888-96.

10 Hankin JH, Wilkens LR, Kolonel LN, Yoshizawa CN Validation of a quantitative diet history method in Hawaii. Am. J. Epidemiol. 1991; 133(6): 616-28.

11 Kemppainen $\mathrm{T}$, Rosendahl A, Nuutinen O, Ebeling $\mathrm{T}$, Pietinen $P$, Uusitupa M. Validation of a short dietary questionnaire and a qualitative fat index for the assessment of fat intake. Eur. J. Clin. Nutr. 1993; 47(11): 765-75.

12 Van Assema P, Brug J, Kok G, Brants H. The reliability and validity of a Dutch questionnaire on fat consumption as a means to rank subjects according to individual fat intake. Eur. J. Cancer Prev. 1992; 1(5): 375-80.

13 Sobell J, Block G, Koslowe P, Tobin J, Andres R. Validation of a retrospective questionnaire assessing diet $10-15$ years ago. Am. J. Epidemiol. 1989; 130(1): 173-87.

14 Jain MG, Howe GR, Rohan T. Dietary assessment in epidemiology: comparison of a food frequency and a diet history questionnaire with a 7-day food record. Am. J. Epidemiol. 1996; 143(8): 953-60.

15 Leighton J, Neugut A, Block G. A comparison of face-to-face food frequency interviews with telephone interviews and self-administered questionnaires. Am. J. Epidemiol. 1988; 128(4): 891.

16 Block G, Hartman AM, Dresser CM, Caroll MD, Gannon J, Gardner L. A data-based approach to diet questionnaire design and testing. A. J. Epidemiol. 1986; 124: 453-69.

17 Willett W. Energy-adjusted method. In: MacMahon B, ed. Nutritional Epidemiology, Vol. 15. Oxford: Oxford University Press, 1990; 261-3.

18 Freedman L. Challenges in statistical approaches to dietary assessment. Eur. J. Clin. Nutr. 1998; 52(2): S6.

19 Caan B, Hiatt BA, Owen AM. Mailed dietary surveys: response rates, error rates, and the effect of omitted food items on nutrient values. Epidemiology 1991; 2(6): 430-6.

\section{Appendix: Food frequency review probes}

1. Verify that a large serving size is $c .1 .5 \times$ medium and adjust frequencies to compensate for very large serving size.

2. Check for discrepancies between the frequency of sugar and milk added to cereals and coffee and the frequency of those items listed. Do the same for margarine and butter on bread and rolls, and salad dressings on salads and sandwiches. 
3. Verify that the foods listed under the restaurant question that are eaten at fast food places or restaurants are included in the main list of food items.

4. Confirm that when combination foods eaten are not listed, that the individual components are included separately.

5. Check for discrepancies between frequency of fruits and vegetables listed in the summary question and total frequency listed across individual items.

6. Add total number of entrée-type items per week to make sure they are in an expected range (7-21 per week).

7. When probing for other fruits, ask about: cherries, cranberry sauce, grapes, kiwi, mango, melon, nectarine, papaya, pineapple, plum.
8. When probing for other vegetables, ask about: artichoke, asparagus, avocado, beet, celery, cucumber, eggplant, mushroom, okra, olive, onion, pepper, radish, sprouts, summer and winter squash, turnips.

9. When probing for meat, fish and poultry, ask about: canadian bacon, lamb, scrapple, tofu, turkey bacon, veal, venison.

10. When probing for mixed dishes, ask about: burritos, chop suey, chow mein, enchiladas, frozen dinners (lite and regular), pasta salad, tacos.

11. When probing for breakfast foods, ask about: egg substitutes, fast food breakfast sandwiches, french toast, granola bars, grits, pancakes, waffles.

12. When probing for breads and grains, ask about: bulgur, corn meal, couscous, hominy, tabouli. 